\title{
Realistic Propagation Simulation of Urban
}

\section{Mesh Networks}

\author{
Vinay Sridhara Stephan Bohacek \\ vsridhara@udel.edu bohacek@udel.edu \\ Department of Electrical and Computer Engineering \\ University of Delaware
}

Newark, DE 19716

\begin{abstract}
Simulation plays an important role in the verification of mobile wireless networking protocols. Recently several cities have either begun deploying or are completing plans to deploy large-scale urban mesh networks (LUMNets). On the other hand, the networking research community has little expertise in simulating such networks. While the protocols are simulated reasonably realistically, the propagation of wireless transmissions and the mobility of nodes are not. Today, simulations typically model propagation with either the free-space model or a "two-ray" model that includes a ground reflection. Such models are only valid in open space where there are no hills and no buildings. Since wireless signals at the frequencies used for mobile wireless networking are partly reflected off of buildings and partly is transmitted into the building, the presence of buildings greatly influences propagation. Consequently, the open-space propagation models are inaccurate in outdoor urban areas. Indoors, the open-space models are not even applicable. This paper presents guidelines for simulating propagation in such urban settings. Furthermore, extensive background discussion on propagation is also included. The techniques for propagation are validated against propagation measurements. The techniques discussed are implemented in a suite of tools that are compatible with protocol simulators and are freely available for use.
\end{abstract}

Stephan Bohacek is the corresponding author. His address is University of Delaware, Dept. of Electrical and Computer Engineering, Newark, DE 19716, USA, 302-831-4274, bohacek@udel.edu. 


\section{INTRODUCTION}

Recently, there has been interest in developing protocols for urban mesh networks such as the ones to be deployed in Philadelphia [1], San Francisco [2], Taipei [3], Minneapolis [4], Anaheim, California, and Tempe, Arizona. Simulation is the common technique to determine the performance of these protocols. However, today, most simulations use the trivial disk propagation model (i.e., the signal propagates exactly $R$ meters and no further) or a highly idealized propagation model such as the free-space or the two-ray model. Due to the presence of buildings, propagation in urban environments is far more complicated than the propagation presented by these simple models. Consequently, channel variability, which is a key aspect of wireless networking, is not well modeled in today's simulations. The result is that many insights gained from the free-space environment do not necessarily hold in the urban environment, casting doubt on the applicability of the conclusions drawn from today's simulations.

While the reasons that realistic propagation models are typically not included into network simulations is not well documented, it seems that the typical reasons include the belief that propagation modeling is computationally intractable and that propagation cannot be realistically modeled due to small-scale fading and delay spread. To the contrary, this paper demonstrates the feasibility of including realistic propagation into network simulation. To this end, a simulator has been developed in accordance with the guidelines set forth in this paper [5]. This simulator is validated in three scenarios and it is shown that when used with packet level simulation, realistic propagation has a dramatic impact on simulation results. Furthermore, while realistic propagation is computationally intensive, the computational complexity of the approach taken here results in the propagation aspects of the typical simulation taking approximately as long as the processing of the discrete events by the packet simulator. Consequently, this paper provides techniques and methods for greatly improving the quality of simulations.

In order to justify the simulation strategy used, an overview of the key features of propagation in an urban environment is presented. This nonmathematical discussion of propagation also provides networking researchers with an intuition of propagation in urban environments. For example, some of the myths that can be found in the networking literature are dispelled, and issues that are neglected in the networking literature, such as the importance of building materials, are discussed.

The remainder of this paper proceeds as follows. In the next section, stochastic models of propagation are discussed. While stochastic models are often used in communication theory, their applicability to networking is 
limited, and, as will be discussed, they should be used with care. In contrast, the propagation simulator discussed here is deterministic. Section III discusses several characteristics of propagation. Specifically, the subsections of Section III discuss transmissions through walls, reflections off of walls, multipath fading, diffraction, scattering, timevarying channels, and delay spread. Section IV discusses computational issues, discusses details of the UDelModels' propagation simulator, and presents validation of the simulator. Section $\mathrm{V}$ provides some discussion on the impact that realistic simulation has on the performance of networking protocols. As one might expect, traditional simulations that use random way-point mobility and free-space propagation yield very different results than the simulations carried out using realistic mobility and propagation. It is important to note that realistic simulation of wireless networks is an ongoing effort and that as the computational capabilities increase, more detailed and accurate simulations are possible. Some areas that will benefit from further effort and improved accuracy are discussed in Section VI. Related work on propagation simulation is provided in Section VII and finally, concluding remarks can be found in Section VIII.

\section{Stochastic Models of Propagation}

One of the most important aspects of propagation is the channel gain (other characteristics are discussed below). The objective of propagation simulation is to determine the channel gain (and other channel characteristics). The physical layer model then uses these channel characteristics to determine the probability of transmission error. The relationship between the received signal power $P_{\text {received }}$ and transmitter signal power $P_{\text {transmitted }}$ is $P_{\text {received }}=$ $K \times H \times P_{\text {transmitter, }}$, where $K$ is a constant that depends on the wavelength and the antennas, and $H$ is the channel gain. It is common for this relationship to be specified in $\mathrm{dB}^{1}$ and $\mathrm{dBm}$ ( $\mathrm{dB}$ milliWatts), i.e.,

$$
P_{\text {received }}[d B m]=10 \log _{10} K+H[d B]+P_{\text {transmitter }}[d B m] .
$$

To get an idea of common values, consider the typical $802.11 \mathrm{~b}$ transmissions. In this case, the transmission power is $15 \mathrm{dBm}$ and $10 \log _{10} K=-41 \mathrm{~dB}$. Furthermore, the sensitivity of commercially available 802.11b receiver cards is approximately $-93 \mathrm{dBm}$ when receiving at $1 \mathrm{Mbps}$. Thus, if the channel gain is lower than $-67 \mathrm{~dB}$, then it is not possible to decode the transmission with marginal reliability. It is also common to require an extra gain

\footnotetext{
${ }^{1}$ For the remainder of the paper, we specify channel gains in $\mathrm{dB}$.
} 
margin to allow for the signal to be decoded reliably (not simply marginally reliability), to account for losses due to connectors and other analogue circuits, and to allow for overly optimistic sensitivity specifications. The gain margin depends on the transmitter/receiver manufacturers and system design, and can range from $3 \mathrm{~dB}$ up to $9 \mathrm{~dB}$, which require the $802.11 \mathrm{~b}$ communication channel gains to be above $-64 \mathrm{~dB}$ and $-58 \mathrm{~dB}$ respectively.

The propagation of wireless signals can be accurately modeled by Maxwell's Equations. However, it is beyond today's computational abilities to simulate large urban regions using these equations. For this reason, there has been extensive research effort on developing techniques that provide computationally tractable estimates of the channel gain and other characteristics. In general, the techniques can be classified into deterministic and stochastic models. Furthermore, they can be divided into site specific approaches (i.e., detailed information about the environment is included into the propagation model) and non-site specific approaches. As discussed next, for simulation of urban mesh networks, deterministic site specific propagation models are appropriate.

We can easily rule-out deterministic non-site specific approaches such as the Okumura-Hata model [6] or COST 231 [7]. Such models provide a relationship between the channel gain and the distance between the receiver and transmitter. However, these models only provide an average behavior and do not model the variability of the channel.

On the other hand, non-site specific stochastic models can account for channel variability and hence are widely used in communication theory. However, there are many pitfalls to stochastic models which often make them not applicable to urban mesh network simulation. Consider the well established lognormal shadowing model [8]. This model specifies that the received signal power obeys

$$
P_{\text {received }}=\frac{K}{d^{\alpha}} \times Y \times P_{\text {transmission }}
$$

where $K$ is a constant, $d$ is the distance between the transmitter and receiver, $\alpha$ is the attenuation factor, $P_{\text {transmission }}$ is the transmitted power, and $Y$ is a lognormally distributed random variable that accounts for shadowing. This model dictates that if a transmitter and receiver are placed at randomly selected locations, then the relationship between distance, transmitted power and received power obeys (1). While the model has been verified through experimentation, the model focuses only on a single link. On the other hand, in networking, the interest is on a large number of links, specifically, the focus is on the graph formed by links. If the relationship between received and transmitted signal strength merely obeys (1), then the graph formed by links would be a type of a random 

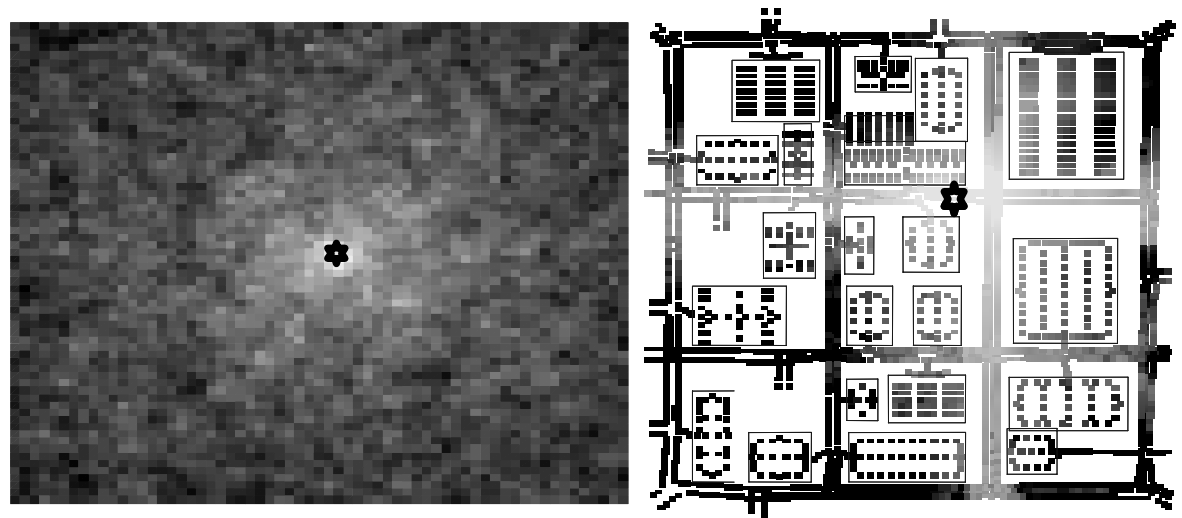

Strongest signal

Weakest signal

Fig. 1. Left, channel gain generated by a correlated lognormal model. Right, channel gains generated by ray-tracing in an urban area. The rectangle shapes are buidlings and the shaded boxes inside the rectangles represent channel gain to locations on the first floor of the building. In both the left and right frames, the star marks the location of the transmitter.

graph, whereas an urban setting induces a graph with deterministic components. Consider the left and right frames in Figure 1. The left-hand frame shows the channel gains (including correlations as described in [9] and [10]) when a lognormal shadow fading model is used, while the right-hand shows the channel gains in an urban area that is found through the techniques described in the next sections. While it is reasonable to assume that even in the urban setting, if two arbitrary locations are selected, then the signal strength will follow the lognormal distribution (i.e., (1) is valid), it is quite clear that channel gain is not random. Rather, the channel gain is greatly influenced by the map of the urban area. For example, the signal propagates far down the streets, while it propagates poorly into or through/over buildings. To see the implication of this, consider the least-hop path shown in Figure 2. In this example, only the first and last hop are able to propagate between indoor and outdoor, while other hops follow streets (outdoor to outdoor propagation). Thus, we see that the propagation results in routes in urban areas that are not random, but are dependant on the structure of the city. For this reason, we do not use non-site specific models, and focus on site-specific models where the layout of the city and buildings influences the propagation. Currently, the UDelModels’ propagation simulator is deterministic. However, future work will include appropriate stochastic elements. 


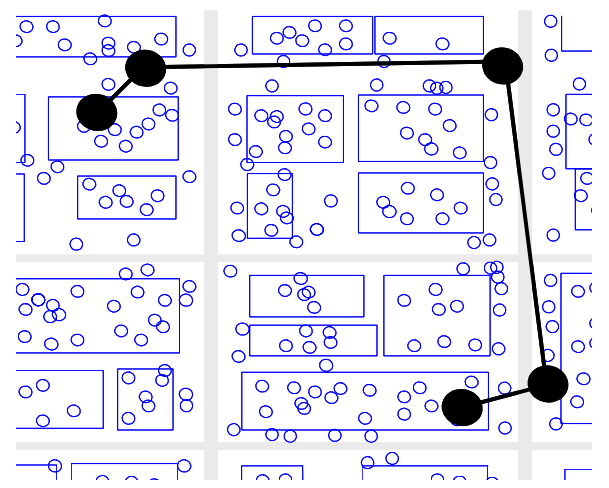

Fig. 2. Routing in an Urban ad hoc Network. The source and destination are inside of two different buildings. The shortest hop path travels along the road, it does not follow the shortest geometric route (i.e., a straight line).

\section{Characteristics of Urban Propagation}

\section{A. Overview}

In the next subsections, several characteristics of propagation are briefly discussed. The objective is to provide intuition of propagation in urban environments. While there are mathematical models of propagation, we do not provide any mathematical details. An excellent reference that contains mathematical details is [11].

Remark 1: We typically focus on the propagation for a particular transmitter and receiver. However, due to reciprocity (e.g. [12]), the channel between two antennas does not depend on which one is the transmitter and which is the receiver. To some degree, the symmetry of channels contradicts the observed asymmetry of links (e.g., [13] and [14]). It is important to note that the observed asymmetry of links is not due to the channel but due to difference in the analogue electronics in the transmitters and receivers. These differences may result in unequal transmit powers and unequal receiver attenuation. In some cases, these differences can be reduced by calibrating the transmit powers. It is also possible that one node experiences more interference than another node. In this case, the probability of error-free packet transmission depends on which node is the transmitter.

\section{B. Transmissions}

In urban mesh networks, reflections off of objects such as buildings and the ground and transmission into buildings play a primary role in the magnitude of the received signal strength. Diffraction and scattering are also important, but from numerical experiments it can be shown that they play a secondary role. When the object that the signal is hitting is much larger than the wavelength, then the behavior of the signal can be accurately modeled as a ray that 
is partially reflected off of the object, partially transmitted through the object, and partially absorbed by the object.

If this ray model is accurate, then there are well known formulas that describe the behavior (e.g., see [11]). In general, the reflection can be written in terms of six components, $H_{\text {Transmit }}^{\perp}\left(\theta_{\text {Incidence }}, \varepsilon_{r}, \varepsilon_{r}^{\prime \prime}, w, f\right), H_{\text {Reflect }}^{\perp}\left(\theta_{\text {Incidence }}, \varepsilon_{r}, \varepsilon_{r}^{\prime \prime}, w, f\right)$, $H_{\text {Absorb }}^{\perp}\left(\theta_{\text {Incidence }}, \varepsilon_{r}, \varepsilon_{r}^{\prime \prime}, w, f\right), H_{\text {Transmit }}^{\|}\left(\theta_{\text {Incidence }}, \varepsilon_{r}, \varepsilon_{r}^{\prime \prime}, w, f\right), H_{\text {Reflect }}^{\|}\left(\theta_{\text {Incidence }}, \varepsilon_{r}, \varepsilon_{r}^{\prime \prime}, w, f\right)$, and $H_{\text {Absorb }}^{\|}\left(\theta_{\text {Incidence }}, \varepsilon_{r}, \varepsilon_{r}^{\prime \prime}, w, f\right)$ where $\theta_{\text {Incidence }}$ is the angle between the ray and the material, $\varepsilon_{r}^{\prime}$ and $\varepsilon_{r}^{\prime \prime}$ are the real and imaginary part of the relative dielectric constant of the material, $w$ is the width of the material, $f$ is the frequency of the signal, and the superscript denotes the polarization.

Instead of detailing how each of these factors impacts the propagation, we will consider an example. We examine the received signal strength on one side of a wall with a transmitter on the other side as shown in left-hand frame of Figure 3. We consider the received signal strength in two locations, specifically, one at the same height as the transmitter and one elevated from the transmitter. We assume that the signal strength decays according to free-space propagation and then intersects the wall and suffers insertion loss $H_{\text {Transmit }}^{\|}$(where we assume that the wireless signal is vertically polarized, hence the $\|$ superscript). We take the frequency to be $2.4 \mathrm{GHz}$, as in $802.11 \mathrm{~b} / \mathrm{g}$. Three types of wall materials are examined, namely, brick $\left(\varepsilon_{r}^{\prime}=3.9, \varepsilon_{r}^{\prime \prime}=0.001, w=10 \mathrm{~cm}\right.$.), concrete $\left(\varepsilon_{r}^{\prime}=5\right.$, $\varepsilon_{r}^{\prime \prime}=0.2, w=20 \mathrm{~cm}$.), and glass $\left(\varepsilon_{r}^{\prime}=5.8, \varepsilon_{r}^{\prime \prime}=0.003, w=2.5 \mathrm{~cm}\right.$.). The behavior for these materials is shown in the right three frames of Figure 3.

Many aspects of transmission through material can be observed in Figure 3. First, notice how in the case where the receiver and transmitter are at the same height, the channel gain increases as the distance between the transmitter and received decreases. This agrees with the intuition from free-space propagation. The impact of the material can be seen by noticing the variation in the received signal strength for different materials. For reference purposes, consider that without the wall, when the transmitter is about $5 \mathrm{~m}$ from the wall, the channel gain is $-14 \mathrm{~dB}$. from the wall and a receiver that is at the same height as the transmitter. Thus, when the angle of incidence is 0 , the wall reduces the channel gain by $0 \mathrm{~dB}$ (for glass) to $6 \mathrm{~dB}$ (for concrete). The impact of the angle of incidence can be emphasized by considering the channel gain when the transmitter and receiver are not at the same height. As can be seen, this results in the signal strength first increasing and then decreasing as the distance between the transmitter and receiver increases; this is in conflict with the behavior in free-space. The key characteristic of propagation at work here is that when the ray hits the wall at a grazing angle, only a small amount of signal power is transmitted 
brick

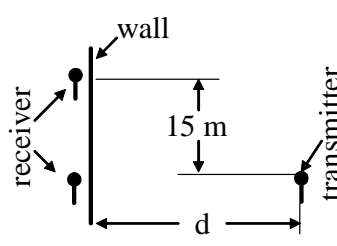

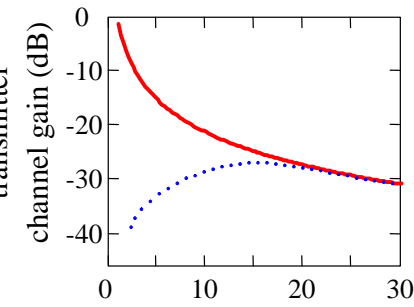

concrete

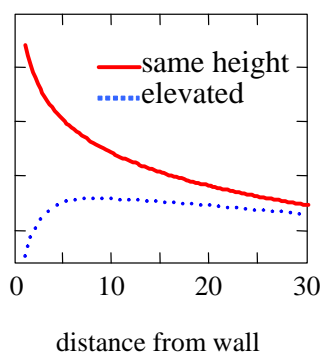

glass

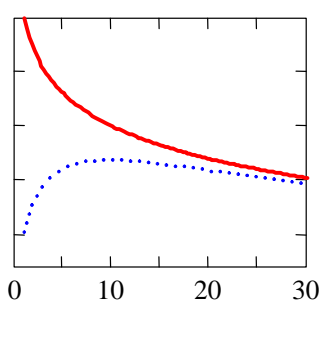

Fig. 3. Transmission through a wall. The figure depicts the attenuation of the signal when transmitted through walls of different kinds of materials. It also depicts the nature of attenuation due to relative heights of the transmitter and the receiver.

through the wall. The point where the signal strength switches from increasing with the transmitter-receiver distance to decreasing with transmitter-receiver distance depends on the material, the thickness of the material, and the height of the receiver.

Remark 2: One interesting implication of Figure 3 is that if an urban mesh network uses relays mounted on lampposts, then the lamppost are quite close to buildings. Thus, relays mounted on a lamppost on the far side of the street will provide better coverage of a building than a relay mounted on a lamppost on the near side of the street. We concluded that, in some cases, the angle of incidence can be more important than the transmitter-receiver distance.

Figure 3 shows variation of the channel gain due to the different building materials. The dependence on material poses a serious challenge for simulation. First, walls are typically not made of a single material, but of layers of material. It is, however, possible to extended the method to accommodate layers of different material (e.g., see [15]) at the expense of computational complexity. A second and more serious problem is that it is not realistic to know the construction material for each building. This dependence on construction materials emphasizes the difficulty of accurate prediction of channel gains and demonstrates why realistic simulation is more reasonable than accurate simulation. In the UDelModels, it is possible to specify different building materials, but it is assumed that the material is homogeneous for each building.

\section{Reflections}

Reflections are complementary to transmissions. However, it is important to note that some power is absorbed by the material, hence, the power transmitted through a wall and the power reflected off of a wall do not necessarily 
sum to the power of the incident signal and, depending on the material and angle of incidence, a significant amount of energy can be absorbed.

To get an understanding of the impact of reflections, we consider propagation down a building lined street, as shown in Figure 4. Here the signal is repeatedly reflected off of walls and, in a sense, focused down the street. This effect is sometimes referred to as propagation down an urban canyon. A similar effect can also arise in hallways and tunnels. The result is that the received signal strength is stronger when propagating down a street than it would be with the same transmitter-receiver distance but in free-space.

Figure 4 shows the channel gain down an urban canyon for the same set of materials considered above and the free-space approximation. Note that free-space predicts a substantially smaller received signal strength than the simulated received signal. For example, when the receiver-transmitter distance is $300 \mathrm{~m}$., the difference between the free-space and the model that accounts for reflection ranges from $13 \mathrm{~dB}$ to $5 \mathrm{~dB}$, depending on the material and the distance between the walls. Figure 4 also shows approximations of the channel gain given by $1 / d^{\alpha}$, where, for a canyon width of $7 \mathrm{~m} ., \alpha=1.38$ and a canyon width of $35 \mathrm{~m} ., \alpha=1.47$. It should be pointed out that it is often assumed that $\alpha \in[2,4]$, however, as this simple example demonstrates, it is possible that for some paths we have $\alpha<2$. That is, while buildings may block communication, they may also enhance communication.

Note the lack of smoothness when the walls are made of brick and the width is $35 \mathrm{~m}$ (e.g. $d=50$ ). This is due to the wide variation in the reflection coefficient as the angle of incidence varies. Indeed, depending on the material and the width of the material, there may be some angles of incidence where no signal is reflected. Such angles are called Brewster angles. In some settings, the wide variation of the reflected signals strength as a function of incident angle results in large fluctuations in the total received signal strength for small movements of the receiver or transmitter antenna. This amounts to further sensitivity of communication on building materials.

\section{Multipath Fading}

It is well known that wireless signals can experience small-scale fading or multipath fading. Such fading results from the constructive and destructive interference of signals that follow different paths from the transmitter to the receiver. As a result, when the wavelength is small, a small displacement of the transmitter or the receiver will cause a change in the interference and hence a change in the received signal strength. While fast-fading is a significant problem in many wireless systems, it is typically not relevant in wide bandwidth communications such as those often 


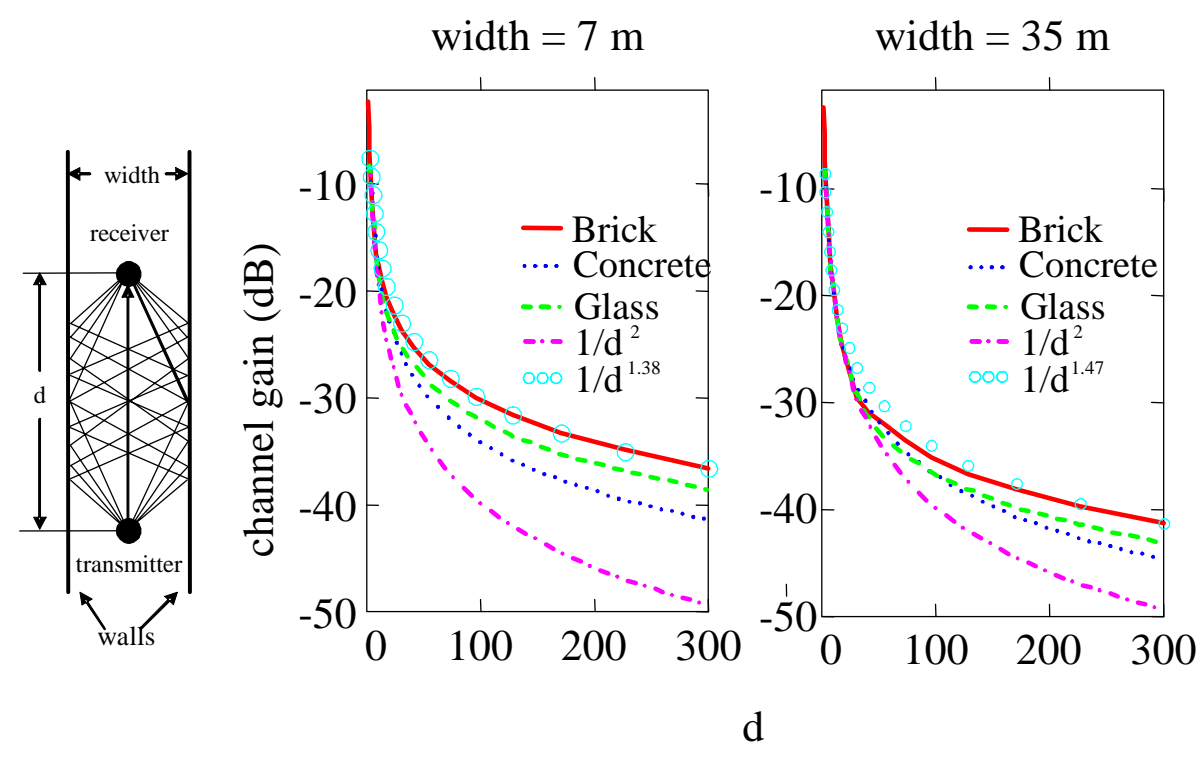

Fig. 4. Propagation down a street. The figure depicts the effect of propagation down an urban canyon for different wall materials, different attenuation exponents, and for the free space

used for data communications (e.g., 802.11). The reason is that the received signal strength is essentially averaged over the bandwidth, which, by definition, is wide. The left-hand frame in Figure 5 shows an example of the signal strength at various frequencies. Note that at some frequencies, the signal strength is quite low, even $40 \mathrm{~dB}$ less than the mean signal strength of $0 \mathrm{~dB}$. A narrow bandwidth communication will experience such degradations in signal strength. However, when averaged over a sufficiently wide bandwidth, the average signal does not experience degradation. For example, the middle frame in Figure 5 shows how the signal strength varies for small changes in position. Note that the narrow bandwidth signal experiences rapid and large fluctuations, while the wide bandwidth case experiences much smaller variations.

In general, the wider the bandwidth, the less susceptible to fast fading. However, variation in signal strength also depends on the environment. The right-hand frame of Figure 5 shows the variance of the received signal strength over a circle with one meter radius as a function of the bandwidth. Each curve is for a slightly different environment, but in all cases it is for propagating down the urban canyon as shown in the left-hand frame of Figure 4. Note that in general, the variance decreases as the bandwidth increases. However, the variance of the narrow bandwidth case and the rate that the variation decreases with the bandwidth depends on the environment. In all cases, the variance is quite small when the bandwidth exceeds $10 \mathrm{MHz}$, while $802.11 \mathrm{~b}$ has a bandwidth of $22 \mathrm{MHz}$. It should be noted that there are other factors besides multipath reflections that could cause large changes in signal strength over small 

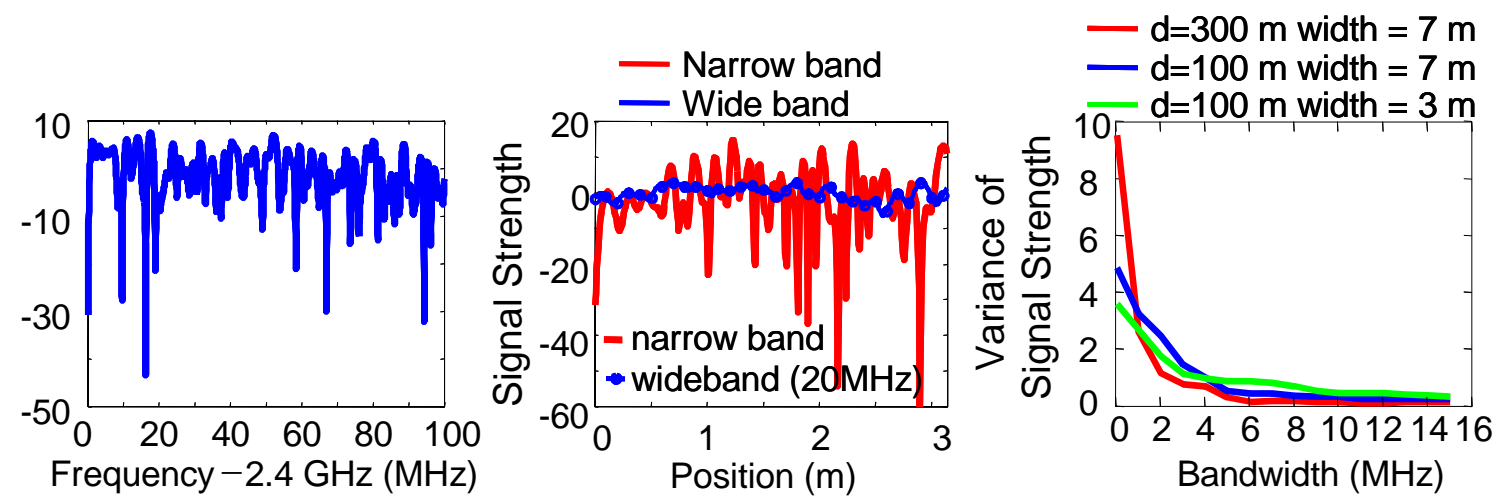

Fig. 5. Fast fading in narrow band and wide band communication scenarios. Left: the signal strength as a function of frequency for a narrow band signal propagating down an urban canyon. Middle: signal strength of a narrow and wide band signal as a function of receiver position perturbation when propagating down an urban canyon. Right: variance in signal strength due to change in receiver position as a function of bandwidth for when propagating down a urban canyon as shown in Figure 4.

changes in position, e.g. changes in antenna orientation.

\section{E. Diffraction}

While reflections and transmission play an important role in propagation, diffraction is also significant and should not be neglected. Diffraction allows the signal to "bend" around corners and over/around buildings. However, the diffracted signal will not be as strong as the original signal; the sharper the bend, the weaker the signal. Consider the examples in Figure 6, which use the Uniform Diffraction Theory [16], [17]. The left-hand frames show the diffraction around a corner. The second from the left frame compares free-space propagation around the corner to free-space propagation with the added loss due to diffraction. The right-hand frames show diffraction over a building where two diffractions are required. In both cases, as $h$ increases, the signal must make a sharper bend (i.e., the diffraction angle increases) and, hence, more loss is incurred. It is important to note that the signal strength decreases quickly as $h$ increases. From the right-hand frames in Figure 6, it can be seen that diffracting over a building that is only $5 \mathrm{~m}$. higher than the transmitter and receiver results in a channel gain that is too small for typical 802.11 communication (recall the discussion in Section II), while diffracting around a single corner (left hand plots of Figure 6) might result in a sufficiently large channel gains for most 802.11 communications. Indeed, our simulations indicate that for 802.11, diffracting around two corners results in loss that is typically too great for 802.11.

While diffracting around two or more corners leads to large losses, 802.11 might be able to communicate 

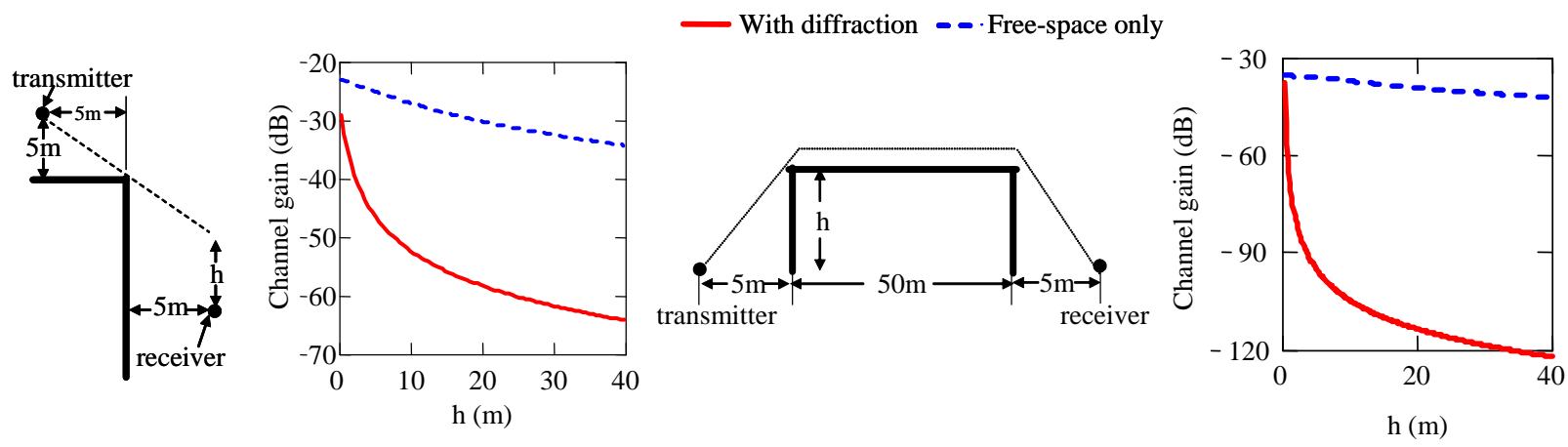

Fig. 6. Diffraction around a corner (left) and over a building (right). As $h$ increases, the signal strength decreases. The dashed lines show the signal strength if the loss was only due to free space propagation while the solid line shows the signal strength when the loss due to diffraction is also included.

effectively around a single corner. Consequently, a significant portion of the coverage area of 802.11 is due to diffraction. Using the UDelModels’ propagation simulator, it is possible to quantify the impact of diffraction. Table I shows the number of locations to which a particular transmitter is able to communicate when only line-of-sight is used, when line-of-sight and reflections/transmissions are used, and when all the components are used, namely, line-of-sight, reflections/transmissions and diffraction. The table also shows the difference in the coverage when different numbers of iterations are used. By the number of iterations, we mean the maximum number of reflections, transmissions, or diffractions that each ray may experience. We see that about $22 \%$ of the locations are missed if diffraction is not used. However, if only line-of-sight is considered, then $78 \%$ of the locations are missed. For these calculations, a map of the Paddington area of London was used and the transmitter and receivers were located $1.5 \mathrm{~m}$ above the ground or above the floor. If the transmitter was higher (e.g., a mobile phone base station), or transmissions with smaller channel gains can be received, then it is possible that diffraction could play a larger role than is illustrated in this example.

\section{F. Scattering}

Scattering is often used to refer to the impact of smaller unmodeled objects on the propagation, e.g., lampposts, trees, vehicles, people, and office furniture. Scattering also accounts for the unevenness of building walls (e.g., windows, doors, or facades). Without detailed knowledge of the location and dimension of small objects and without details of building walls, it is difficult to include the effects of these types of scatterers into propagation simulation. Furthermore, the inclusion of such details greatly increases the computational complexity of propagation 
TABLE I

COVERAge AND COMPUTATION TIME

\begin{tabular}{|l|c|c|}
\hline Experiment & Coverage & Time (sec/transmit location) \\
\hline Line of sight & 937 & 56 \\
\hline 1 iteration & 2623 & 59 \\
\hline 1 iteration without diffraction & 1960 & 56 \\
\hline 2 iterations & 3927 & 62 \\
\hline 2 iterations without diffraction & 2616 & 57 \\
\hline 3 iterations & 4243 & 67 \\
\hline 3 iterations without diffraction & 2862 & 58 \\
\hline 4 iterations & 4265 & 85 \\
\hline 4 iterations without diffraction & 3065 & 63 \\
\hline All iterations with diffraction & 4265 & 122 \\
\hline
\end{tabular}

simulation. As a result, relatively little work has focused on including scattering into propagation prediction. One exception is [18] where the impact of scatterers was examined. It was found that in dense urban environments, such scattering does not dominate. More specifically, at short distances, scatterers have little impact, but may have a some impact at greater distances (e.g., in some cases the difference between prediction and measurements decreased from $6 \mathrm{~dB}$ to $3 \mathrm{~dB}$ when scatterers were included). On the other hand, in areas where building density is low (e.g., suburban areas), scatterers such as lampposts, tress, and vehicles may dominate the propagation. This is one reason why this paper focuses on the urban setting.

Scattering can also occur when a wireless signal propagates through vegetation. Indeed, if the vegetation is large and dense, the scattered signal dominates over the direct, non-scattered signal. Even when the vegetation is thin, as it usually is in urban areas, the vegetation can cause loss. At $2.4 \mathrm{GHz}$, vegetation causes a loss of approximately $0.2 \mathrm{~dB}$ per meter of vegetation [19]. It is also common to model diffraction over vegetation [20].

\section{G. Time-Varying Channel Gain}

While the variability of channel gain is greatly affected by the movement of the transmitter or receiver, the channel gain can also change when the transmitter and receiver are fixed, but objects in the environment move. The right-hand frame of Figure 7 shows the channel gain as a function of time for a receiver and transmitter as observed along a sidewalk during rush-hour in the city center of Philadelphia. As shown in the left-hand frame of Figure 7, the transmitter was $5.4 \mathrm{~m}$ above the sidewalk, while the receiver was only $1.2 \mathrm{~m}$ above the sidewalk. While time-varying propagation is difficult to model, in [21], a diffusion-based model was developed that accurately models the variability shown in Figure 7. However, more work is required to develop models in general settings. 


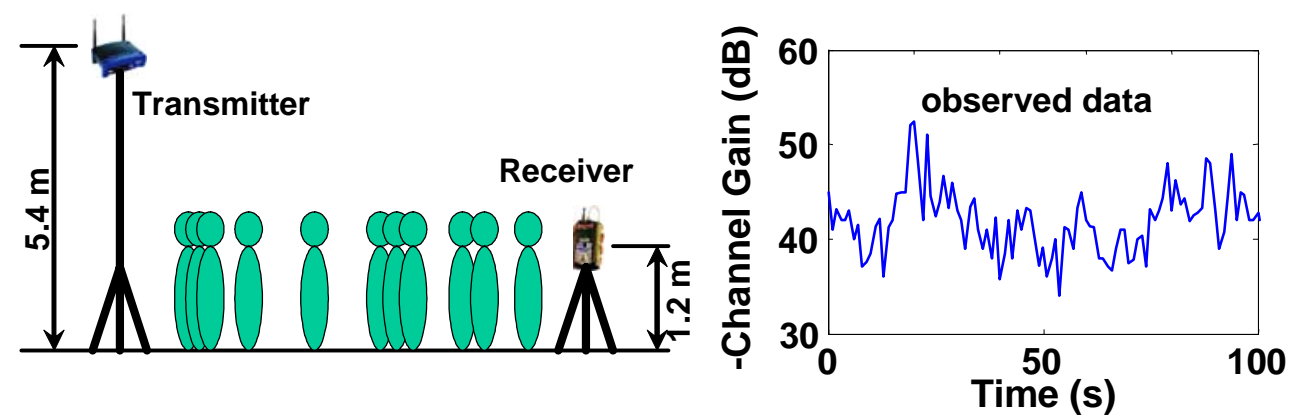

Fig. 7. Left, experimental setup for measuring the time varying nature of propagation in a busy street in Philadelphia. Right, an observed time-series of the channel gain.

It should be noted that the time-varying propagation is due to mobility in the environment.

\section{H. Delay Spread}

As noted in sections III-B, III-C and III-E, the wireless signal may follow several different paths from the transmitter to the receiver. While one impact of multiple paths is that the signal may experience multipath fading, another result is that the multiple copies of the wireless transmission will be received at different points of time. Essentially, these multiple copies will interfere with each other. When this self-interference is considered as noise, it is clear that the effective SNR is decreased by the presence of these delayed copies. Since the traditional calculation of SNR considers delayed copies of the transmission as useful signal power, the traditional SNR might not be a good indicator of probability of transmission error.

A channel where multiple copies of the signal arrive at different times is said to have delay-spread. While there is no ideal metric of delay-spread, two common measures are the mean delay spread and the RMS delay spread (see [8] page 199 for definitions). To explore the impact of delay-spread, consider Figure 8, which shows the relationship between SNR and bit-error probability for different amounts of delay spread for a simple 802.11b receiver and for a 802.11a receiver. Note that the bit-error probability of $802.11 \mathrm{~b}$ is greatly affected by the delay-spread. Also, it is can be seen that for an RMS delay-spread of above 60 ns., which is quite common in an urban setting, the impact of the delay is quite severe. From measurements, it has been found that indoors, the RMS delay-spread is typically less than 50 ns., but outdoors, it can exceed 500 ns. The dependence of delay-spread on the communication environment has been widely observed (e.g, [13] and [22]). It should be noted that the delay-spread typically increases with the distance between the transmitter and receiver [23]. And therefore, roof-top configurations such as those studied 
in [13], which are able to propagate considerable distances due to the lack of obstructions at higher heights, are likely to experience large delay-spread values. As pointed out in [13], to predict the transmission error probability, in some cases, both delay-spread and SNR are required.

Incorporating the effects of delay-spread into wireless simulation poses significant difficulties. First, the mapping from RMS or mean delay-spread to transmission error probability is not well defined. It is possible to have a channel with high RMS delay-spread that yields lower transmission error probability than the one that has a lower RMS delay-spread and conversely. Second, RAKE receivers can be incorporated into receivers that can greatly mitigate the effects of delay-spread. However, the implementation of the RAKE receiver has a significant impact on the performance. For example, [24] shows that high performance RAKE receivers can mitigate the impact of delay-spread, while less effective RAKE receivers will perform poorly when the delay-spread is large. Thus, it is difficult to make any conclusions about the effect of delay-spread on future mesh networks based on current receivers.

In anticipation of receivers more suitable for outdoor use, another alternative is to neglect delay-spread. Not only is such an approach reasonable when the inclusion of algorithms that mitigate the effect of delay-spread into 802.11b receivers is anticipated, but also because other physical layers based on OFDM greatly mitigate the impact of delayspread. Consider Figure 8, which shows the relationship between SNR and bit-error probability for different values of RMS delay-spread when 802.11a is used. Note that the impact of delay-spread is not discernible. Schemes such as $802.11 \mathrm{~g}$ and 802.16 use OFDM and hence are also relatively immune to delay-spread. In summary, while delayspread can have a significant impact on transmission error, there are a number of physical layer schemes that can mitigate these effects, and, considering the rapid migration between physical layers (e.g., from 802.11b to 802.11g), it seems likely that when there is a need (i.e., when outdoor mesh networks are finally deployed) such schemes will become wide-spread. Therefore, depending on the assumptions made about the network being simulated, realistic simulation of future urban mesh networks does not necessarily require consideration of delay-spread.

\section{Computational Techniques}

\section{A. Overview}

While much is known about propagation, a major obstacle to simulation of propagation is the computational complexity. This complexity forces a trade-off between computational complexity and accuracy. The difficulty to 


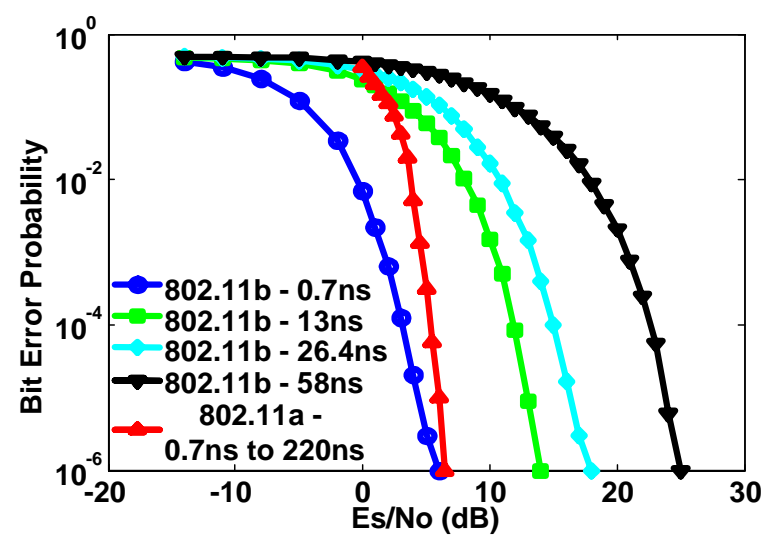

Fig. 8. Bit-error probabilities as a function of delay-spread and SNR. Simulation of 802.11b at 1 Mbps (without a RAKE receiver) and 802.11a at 6 Mbps. The different curves for 802.11b correspond to different RMS-delay spreads. For a delay-spread of 7ns to $220 \mathrm{ns,} 802.11 \mathrm{a}$ gave the same relationship between SNR and bit-error rate, hence this relationship is shown with a single curve.

specify the details of the environment (e.g., wall materials and exact dimensions and structure of buildings) also forces a trade-off with accuracy. It is important to note that the propagation must be determined from every location that a transmitter/receiver could be located to every other possible location that a transmitter/receiver could be located. The simulation of a $1 \mathrm{~km} \times 1 \mathrm{~km}$ urban region often requires hundreds of thousands of locations. Hence, the computation to determine the signal strength from a single location, which is in itself computationally difficult, must be repeated hundred of thousands of times. In this section, techniques to efficiently simulate propagation are discussed. The techniques described are part of the UDelModels simulation package. In the next subsection, outdoor propagation is discussed, and is followed by a discussion on indoor propagation simulation.

\section{B. Outdoor Propagation}

Once the map of buildings, bandwidth, and building materials have been defined, propagation can be estimated. As noted above, extreme care must be taken to reduce the computation. A significant computational savings can be achieved if it is assumed that all walls are vertical. In this case, the 3-D ray tracing problem reduces to a two stage problem, each of which are much smaller than the 3-D problem. The first stage is illustrated in the left-hand plot in Figure 9, where two vertical plane paths are shown (the path that diffracts around the smaller building is not shown). The first stage is a 2-D ray-tracing. Once the vertical plane paths are found, the 3-D ray paths restricted to the vertical plane paths can easily be computed. The right-hand frame in Figure 9 shows the paths of rays in the vertical planes. One vertical plane path has three ray paths, one that diffracts over a building, one 

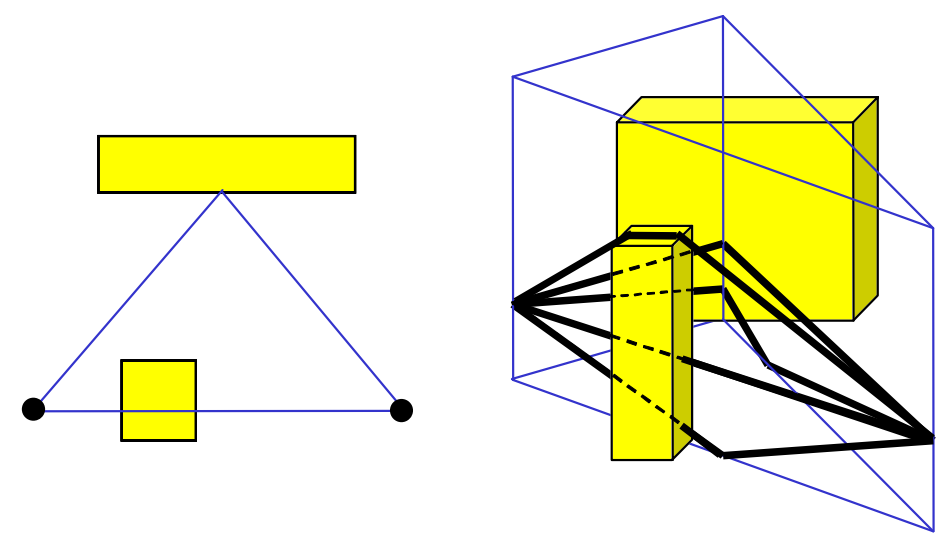

Fig. 9. Left: a top-view of the scene on the right. The lines shown are found from 2-D ray-tracing. Right:: Two vertical plane paths (found from 2-D ray-tracing) and 5 ray paths.

that reflects off of the ground and passes through a building, and one that passes straight through a building. The other vertical plane path has two ray paths, one reflecting off of the wall of a building and one reflecting off of the wall of building and undergoing a ground reflection. While Figure 9 shows each vertical plane paths having two or three ray paths, in general, in one vertical plane path there are many ray paths that may include reflection off of the ground, and transmissions through buildings or diffractions over buildings. The UDelModels' propagation simulator only considers three types of ray paths, direct paths (no ground reflections and no diffractions over buildings), ground reflected paths with no diffraction, and paths that include no transmissions through buildings but rather diffract over buildings. Note that diffractions and transmissions through buildings result in significant loss, and hence neglecting ray paths with multiple diffractions or diffractions and transmissions into buildings has little impact on accuracy. This method of 3-D ray-tracing is known as vertical plane launching [25], and is known to yield accurate propagation estimates in real cities, where all walls are not necessarily vertical [26].

A straightforward implementation of even 2-D ray-tracing is computationally difficult. Instead, a technique that is more appropriately called beam tracing can be performed. Like ray tracing, the goal of beam tracing is to determine the paths from the transmitter to receiver. Beam tracing begins with the source broadcasting the signal in all directions. This transmission is not modeled as a large number of rays, but as a small number of beams. When a beam intersects a building, two beams are generated, one is reflected off of the building and one is transmitted into the building. If only a part of the beam intersects the building, then multiple beams are generated, in particular, one that reflects off of the building, one that transmits into the building, one that passes by the building, as well 
as several beams that account for the diffracted signal. Each diffracted beam models a particular range of angles of diffraction. The strength of the diffracted beam is the average strength for the diffraction angles that the beam models. Note that smaller the range of angles for each beam, the more accurate the diffraction model is. But this generates more beams, increasing the computational complexity of the process. Finally, if the receiver is found to be included within the span of a beam, the vertical plane path is found and the 3-D rays from transmitter to receiver are determined. Once a 3-D ray is found, the 3-D ray information, specifically, signal strength, phase, delay, angle of departure from the transmitter, and angle of arrival at the receiver are recorded.

The beam tracing computation can be further simplified by dividing the 2-D space into a grid and the determining the propagation between the center points of each square. Each square of the grid is called a floor-tile. Outdoors and indoors are discretized in this manner. To reduce the number of floor tiles, the entire space is not discretized. Rather, floor-tiles are placed only along the center-lines of sidewalks, roads, and hallways. For rooms, floor tiles are placed in every location that a mobile node can be located. Similarly, the walls of buildings are also divided into wall-tiles. Since the beam tracing is in 2-D, the wall-tiles are segments (1-D tiles). The smaller the size of these tiles (floor-tiles and wall-tiles) more accurate the simulation results, but also the more computationally expensive.

The computation is divided into two parts, preprocessing and beam tracing. During preprocessing, ray neighbors for each tile are found. A tile's ray neighbors are all the tiles that could be directly reached (i.e., without reflection, transmission through a wall, or diffraction) by a ray emanating from the tile. Once the ray neighbors are found, beam tracing can be performed efficiently. Figure 10 illustrates how the beam tracing with wall tiles is performed.

The process of beam tracing Figure 10 is carried out in a breadth first manner ${ }^{2}$ with each beam continued to be reflected, transmitted until either the beam exits the modeled area or until the estimated channel gain of the beam falls below a threshold. Each time a beam is generated, the floor-tiles it covers are determined and the 3-D ray information is recorded. Note that the 3-D ray information allows one to interpolate the signal strength between grid points as well as determine the delay-spread, the Doppler spread (once velocity is known), as well as simulate directional antennas. It is possible to determine the receiver power over a particular bandwidth by integrating the signal strengths in a way that accounts for phase (i.e., accounting for constructive/destructive interference). The 3-D ray information requires a significant amount of information be saved. A considerable amount of savings is

\footnotetext{
${ }^{2}$ Here, breadth first means that all the $k$ th reflections are found before the $(k+1)$ th reflections are found.
} 


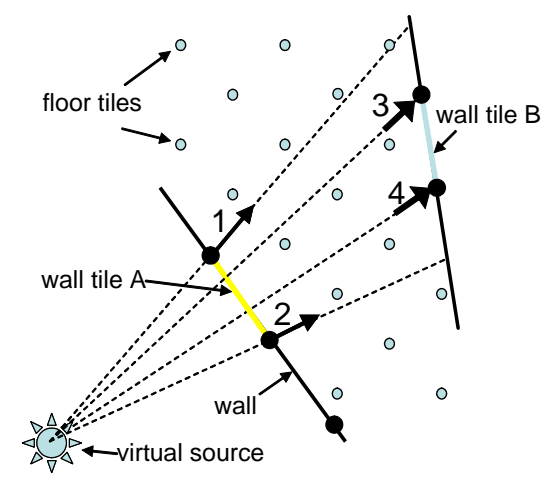

Fig. 10. Beam Tracing. Suppose a beam strikes the wall tile marked A. The reflection of this beam is modeled as a beam emanating from the virtual source as shown. This beam is between and is defined by the two rays marked 1 and 2 . These rays are used to select the floor tile ray-neighbors that this beam strikes (since ray-neighbors are precomputed, this selection is computationally efficient). Similarly, the rays 1 and 2 are used to select the set of A's wall tile ray-neighbors that the beam strikes. One such wall tile is shown in the upper right and is marked B. The beam that strikes tile B is defined by the rays marked 3 and 4 . These rays define the beam that is transmitted through tile B and can be used to find the beam that is reflected off of $B$. This process of reflection and transmission is repeated until the beam's strength is small enough that it can be neglected (e.g., $-110 \mathrm{~dB}$ ).

achieved by simply recording the received power over a bandwidth and RMS delay-spread (which is merely two integers). Of course, this approach cannot be used if directional antennas are simulated or Doppler spread included.

Another important computational saving is achieved by recognizing that the communication is not possible between most locations, hence the propagation matrix is sparse. Computational savings are possible by using techniques to store sparse matrices. However, since the resulting data is large, and since simulation requires the determination of a large number of channel gains, it is important that the sparse matrix techniques support fast access.

\section{Indoor Propagation}

Beam tracing can be performed indoors as well as outdoors. However, the computational complexity depends on the number of walls. Since building interiors have a large number of walls, beam tracing inside all the buildings within a large region of a city exceeds today's computational abilities. Fortunately, it has been found that a realistic estimate of indoor propagation can be performed without using beam tracing. Specifically, the attenuation factor (AF) model has been shown to provide realistic channel gain estimates, with the error found to be within $4 \mathrm{~dB}$ [8]. The AF model assumes that communication indoors takes a straight line path (i.e., no reflections off of interior walls). Furthermore, transmissions through each interior wall and transmissions through each floor result in attenuation. While the amount of attenuation depends on the building, a value of $4 \mathrm{~dB}$ per wall (for an office building) has been shown to work well [8]. Realistic attenuation for passing through 1 floor is $30 \mathrm{~dB}$, passing through 2 floors is $35 \mathrm{~dB}$, 
passing through 3 floors is $39 \mathrm{~dB}$ and passing through 4 or more floors is $40 \mathrm{~dB}$ [8]. In summary, outdoors, rays make reflections off of buildings, diffractions over and around buildings, and transmissions into buildings. Once inside a building, the ray will continue in the same direction, experiencing further attenuation for any interior wall or floor that it passes through. When a ray strikes an exterior wall from the inside, it is both reflected back inside and transmitted outside.

\section{Computational Complexity}

Determining the propagation matrix of a region of an urban area is a feasible but highly computationally complex task. The complexity is both in terms of memory usage and processing time. Processing times for a $1 \mathrm{~km} \times 1 \mathrm{~km}$ urban region is often on the order of tens of processor days. But the process is highly parallelizable and nearly scales with the number of processors used (i.e., 75 processor days takes about 5 days on 15 processors). Of course, the entire channel gain matrix for each city only needs to be found once. Table II outlines the memory requirements and the processing time for two cities. These two cities represent two ends of the spectrum. Paddington is a dense city with relatively small buildings, whereas the University of Delaware campus has much more open space and larger buildings. Note that the campus map is significantly larger than Paddington, but only has slightly more buildings.

The memory requirement is dominated by the lists of ray neighbors. In Table II, the wall tiles were 2 meters long and floor tiles were $1 \mathrm{~m} \times 1 \mathrm{~m}$. While the number of wall and floor tiles scales linearly with the reciprocal of the size of the tiles (i.e., if the wall tiles are twice as long, there are half as many) ${ }^{3}$, the total ray neighbors scale quadratically. For the simulations shown in Table II, there are between 20,000 to 40,000 exterior floor tiles, and 80,000 to 100,000 interior floor tiles. As expected, sidewalks and roads utilize little area outdoors, but indoors, hallways and offices fill large areas.

As shown in Table II, there are on the order of 10 to 100 million ray neighbors for a city of size about $1 \mathrm{~km} \times 1 \mathrm{~km}$. Since each list entry requires 40B, the memory requirement approaches 4GB. Assuming sufficient memory resources, the computation time for the preprocessing stage is relatively short; the cities shown in Table II took a single day on an AMD Athlon 64 FX 55 with 8GB RAM.

Once the ray neighbors are found, the propagation characteristics between each pair of floor tiles can be found. From a single source, the propagation characteristics to all destinations can be found at the same time. Table

\footnotetext{
${ }^{3}$ Recall that the floor tiles cover sidewalks, roads, hallways, and offices only.
} 
TABLE II

COMPUTATIONAL COMPLEXITY

\begin{tabular}{|c|c|c|c|c|c|c|c|c|c|}
\hline City & Size (m) & $\begin{array}{c}\text { Num } \\
\text { buildings }\end{array}$ & $\begin{array}{c}\text { Num } \\
\text { floor tiles }\end{array}$ & $\begin{array}{c}\text { Num } \\
\text { wall tiles }\end{array}$ & $\begin{array}{c}\text { Total } \\
\text { num. ray } \\
\text { neighbors }\end{array}$ & $\begin{array}{c}\text { Ave. num } \\
\text { reflections } \\
\text { per source }\end{array}$ & $\begin{array}{c}\text { Ave. num } \\
\text { diffractions } \\
\text { per source }\end{array}$ & $\begin{array}{c}\text { Ave. num } \\
\text { vertical plane } \\
\text { paths/source }\end{array}$ & $\begin{array}{c}\text { Total } \\
\text { comp. time } \\
\text { (processor days) }\end{array}$ \\
\hline Paddington & $857 \times 836$ & 131 & $108 \mathrm{~K}$ & $17 \mathrm{~K}$ & $15 \mathrm{M}$ & $194 \mathrm{~K}$ & $367 \mathrm{~K}$ & $1.3 \mathrm{M}$ & 53 \\
\hline UD Campus & $1768 \times 1597$ & 151 & $147 \mathrm{~K}$ & $19 \mathrm{~K}$ & $67 \mathrm{M}$ & $84 \mathrm{~K}$ & $332 \mathrm{~K}$ & $946 \mathrm{~K}$ & 72 \\
\hline
\end{tabular}

II shows that each source produces vertical planes that make approximately 500,000 reflections, diffractions, or transmissions. These reflections, diffractions, or transmissions are shared among all destinations. However, for each destination, all the vertical plane paths between the source floor tile and destination floor tile are found. Hence, the total number of vertical plane paths found greatly exceeds the total number of reflections, diffractions, and transmissions.

For each source it is necessary to find the all reflections. Thus, in the campus map, the total number of reflections found was around 263 billion and 12 billion for Paddington. Several optimizations and tricks to efficiently distribute the program results in the total processing time shown.

\section{E. Validation}

The goal of the propagation simulation for simulating mobile wireless networks is not to predict the signal strength, but to produce signal strength that behaves in a realistic fashion. Nonetheless, it is useful to understand the accuracy of the propagation simulation. To this end, three experiments were performed, two outside and one inside. In all cases, an $802.11 \mathrm{~b}$ access point was placed on a 1.5 meter tripod and the Berkeley Varitronics Yellowjacket wireless receiver [27] was placed on a second 1.5 meter tripod. The access point was placed at a fixed location and the average receiver signal strength was determined by receiving $600802.11 \mathrm{~b}$ beacons. Figure 11 (b) shows a part of the University of Delaware campus and Figure 12 (b) shows a street intersection in Philadelphia. In Figure 11 (b), the buildings were approximately 14 meters high while in Figure 12 (b) the buildings were at least 40 meters high. In both cases, the $\mathrm{X}$-mark denotes the location of the transmitter while the receiver is moved along the indicated path. Figure 11 (a) shows the observed and modeled channel gain corresponding to the path starting from the transmission point and moving along the path in the counter-clockwise direction. Figure 12 (a) shows the model and observed channel gain starting near to the transmitter, moving down and then turning the corner.

Figures 11 (a) and 12 (a) show that the model and observations match well both qualitatively and quantitatively 
(within $5 \mathrm{~dB}$ in most locations). To gain more insight into propagation modeling we examine the propagation prediction quality at different locations, especially the location where the prediction quality is low. In the area between B and C, there is an unmodeled archway that is indicated in Figure 11 (b). Similarly at location F, there is a bridge as depicted with the rectangle. Ignoring these objects impacts the accuracy of the propagation prediction. In the locations marked E and G, there are several moderate sized unmodeled objects (large air conditioners at E and trees at G) that partially blocked the signal. As mentioned above, sometimes such objects are called scatterers. We see that scatterers can slightly decrease the received signal strength. On the other hand, in the areas where there is purely line-of-sight (marked As), line-of-sight with reflections (marked Bs) and reflections with diffraction (marked D), there is good agreement between the model and the observations. See [28] for further discussion on the relationship between accuracy of propagation prediction and accuracy of the map. Furthermore, it is important to note that due to mobility of objects, propagation measurements made at different times will be different. For example, in [29] it was found that identical measurements made at different times can differ by $5 \mathrm{~dB}$.

Figure 12 (a) also shows a good fit. Again, the influence of scatters can be observed. In this case the scatterers includes things such as mailboxes, parked cars, and irregularity of the walls (e.g., doors that are set back from the wall). Nonetheless, the model and observations are within a few $\mathrm{dB}$. Note that the signal strength decreases quite slowly as the distance from the corner increases. This is due to the urban canyon effect discussed in Section III-C and depicted in Figure 4.

Finally, Figure 13 (b) shows the layout of a building interior and Figure 13 (a) compares the modeled and observed signal strength for the points indicated in Figure 13 (b). Again, we see that there is reasonable good agreement between the model and the observations.

In summary, it is clear that accurate propagation prediction requires more detailed knowledge of the environment. However, coarse knowledge (e.g., location of buildings alone) provides realistic propagation, both qualitatively and quantitatively. 


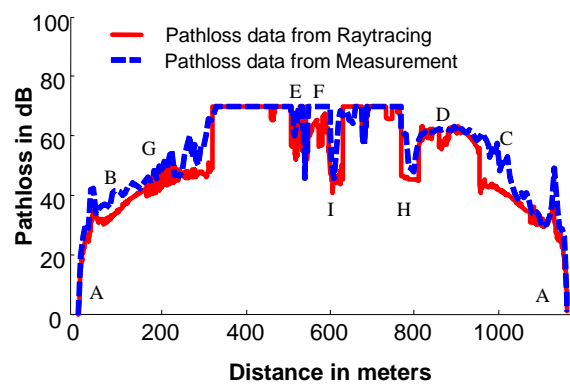

(a)

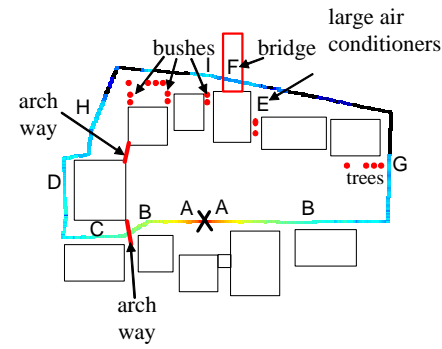

(b)

Fig. 11. Observed and estimated path loss in the campus environment.

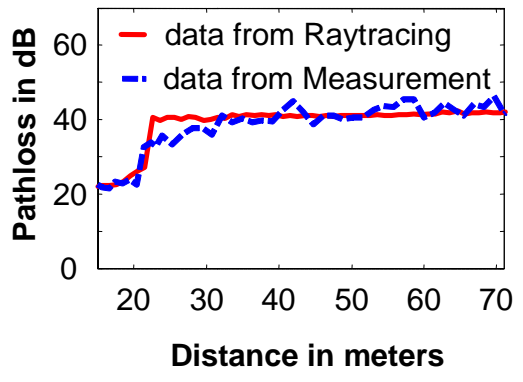

(a)

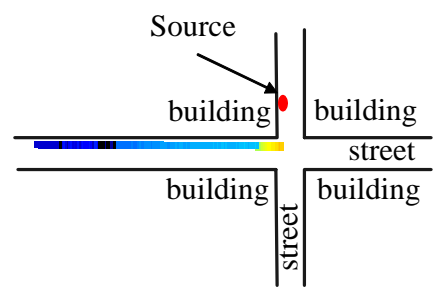

(b)

Fig. 12. Observed and estimated path loss at an intersection in Philadelphia.

\section{IMPACT ON MOBile WiRElEss Simulations}

In much of the mobile wireless networking literature, random way-point with open-space ${ }^{4}$ propagation (RWP/OS) are used. It is clear that this simple propagation models is quite different from the one discussed here. A natural question is how the simulation strategy impacts the conclusions drawn from the simulations? However, a direct comparison of open-space propagation and realistic urban propagation is difficult. One problem is that random way-point mobility makes little sense in urban area. For example, since urban areas are in three dimensions, a random way-point model would have nodes flying through the air between buildings. Hence, in order to perform sensible simulation, an urban mobility model is necessary. To this end, the UDel Urban Mobility Model is used [30].

Another problem with comparing open-space propagation to realistic urban propagation is that the resulting networks are very different and a hence meaningful comparison is difficult. For example, in an open-space environment,

\footnotetext{
${ }^{4}$ By open space, we mean the 2-ray model, where the received signal power decays as $C_{2}^{2} / d^{2}$ for $d<200 m$ and as $C_{4} / d^{4}$ for $d \geq 200 m$, where $C_{2}$ and $C_{4}$ are constants.
} 


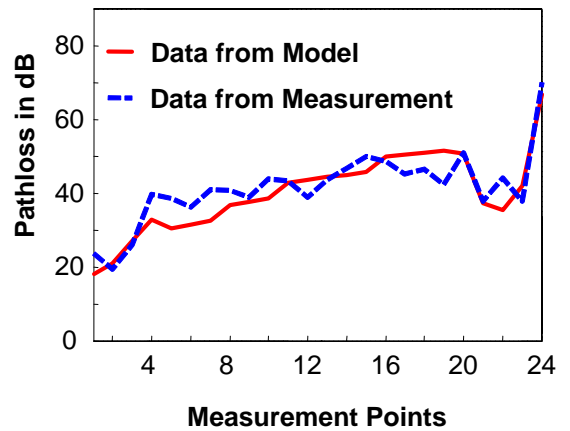

(a)

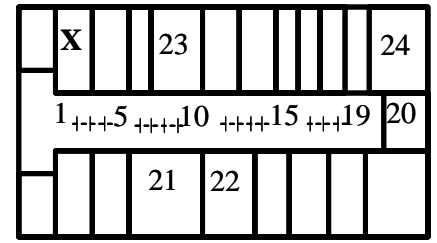

(b)

Fig. 13. Observed and estimated path loss in an indoor environment. The measurement points shown on the x-axis correspond to the numbered locations in the map. The map is of the third floor of Evans Hall in University of Delaware campus.

distant nodes are able to communicate whereas in an urban setting, the nodes separated by very small distances may not be able to communicate (e.g. when one of the communicating nodes is indoors and the other is outdoors), or, as shown in Figure 4, nodes that are far apart may be able to communicate (e.g., when communicating down an urban canyon). To see the impact of the variability in propagation, consider Figure 14. These plots show the mean number of nodes that a node can communicate with (i.e., the mean degree) and the coefficient of variance (CoV) of the degree. These plots show are from three scenarios, namely, urban mobility with realistic urban propagation, urban mobility with open-space propagation, random way-point mobility with open-space propagation. In all cases, the simulated region is generated from a 9 block $(\sim 0.5 k m \times 0.5 k m)$ region of Chicago. The case of random way-point also uses the Chicago map, but nodes move according to the random way-point mobility and propagation is given by the 2-ray model. While the random way-point mobility is distinct from urban mobility, we selected the node speeds to be similar, i.e., Gaussian with mean $1.34 \mathrm{~m} / \mathrm{s}$ and standard deviation 0.26 [30]. In all cases, a mesh infrastructure was placed on 29 uniformly distributed streetlights. The statistics of the degree distribution of the infrastructure of the mesh and the mobile client nodes are shown for several different numbers of mobile nodes in the network. It can be seen that the degree distributions are different in a number of ways. The most obvious difference is that the open-space propagation results in a much larger mean degree. On the other hand, the coefficient of variance is smaller, indicating less variation in the degree, i.e., the degree is more stable in openspace propagation environment than in the urban environment. Furthermore, comparing the statistics of the degree distribution of the infrastructure nodes to the client nodes, we see that the urban setting shows a larger difference between these types of nodes than does the open-space. 


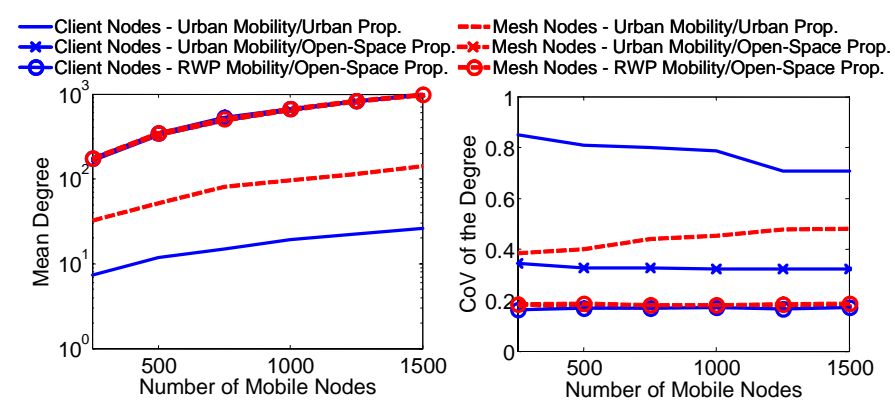

Fig. 14. Statistics of degree distribution in random way-point with open-space propagation, urban mobility with open-space propagation and urban mobility with realistic urban propagation. The mesh infrastructure nodes are fixed on top of streetlights, while the client nodes are mobile. The coefficient of variance $(\mathrm{CoV})$ is the standard deviation divided by the mean.

To further examine the differences between the realistic propagation and open-space propagation, consider the plots shown in Figure 15. These performance statistics are also generated from the 9-block region of Chicago as described above. Twenty UDP connections were initiated from a centrally located base station to randomly selected client nodes. Each connection attempted to send a packet once every 250 msec. An adapted version of AODV [31] was used. Specifically, when a mobile node receives a route request (RREQ) from an infrastructure node, the mobile node will not relay the RREQ until 100 msec. has past. In this way, routes tend to follow the infrastructure, but mobile-to-mobile relaying is still possible. Three environments were considered, namely, urban mobility with urban propagation, urban mobility with open-space propagation, and random way-point with open-space propagation.

As expected, Figure 15 shows dramatic difference between urban propagation and open-space propagation. As alluded to above, realistic node densities for urban environments result in very high densities when open-space propagation is used. Such high densities result in a large number of collisions which lead to route searches failing and even route failures. Indeed, for 750 or more mobile nodes, very few packets are successfully delivered in the open-space propagation setting. Note that there is little difference between urban mobility with open-space propagation and random way-point mobility with open-space propagation. However, this does not indicate that mobility is unimportant, but indicates that mobility is unimportant when used with open-space propagation. The impact of mobility models when realistic propagation is employed is beyond the scope of this paper.

As discussed in Section IV-D, determining the propagation matrix is computationally intensive, however, it only must be done once for each city. Once the propagation matrix is found and a mobility model determines the locations of nodes, it is necessary to select the entries from the propagation matrix corresponding to each pair of node locations. This channel information is saved into a propagation trace file, which the protocol simulator reads 

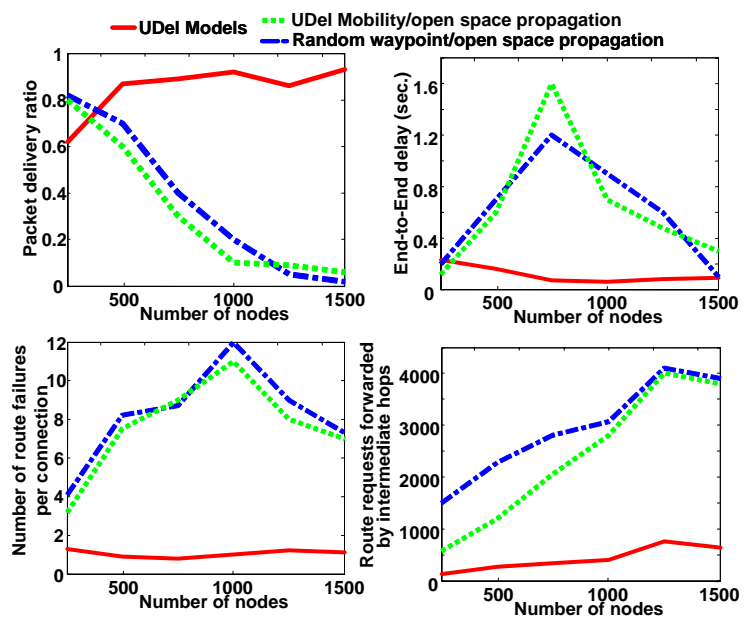

Fig. 15. Performance of a network under differrent types of mobility and propagation.

during packet simulation. Figure 16 shows the execution time, which includes the time to construct the propagation trace file and the time to run the packet simulation for a one minute of simulated time. Figure 16 demonstrates that realistic propagation does increase the execution time. This increase is less than a factor of two for high node densities, but is quite large for low node densities. On the other hand, the right-hand frame in Figure 16 shows that for 250 mobile nodes, the packet simulation time makes up the majority of the execution time, and at all node densities, the time to construct the propagation trace file is similar to the packet simulation time. Furthermore, since the time to perform a packet simulation depends on the number of discrete events that must be processed, the propagation environment will have a significant impact on the packet simulation time. Hence, comparing packet simulation times of open-space propagation and realistic propagation is difficult.

While Figure 16 shows that the time it takes to perform packet simulation is similar to the time it takes to generate the propagation trace file, it is possible to generate the propagation trace file once and reuse the same trace file for multiple simulations. In fact, a large set of propagation trace files are available online [5]. If this approach is used, then the only computation related to realistic propagation is loading the propagation trace file. However, since the trace file specifies the propagation between all pairs of nodes, if a large number of nodes are simulated, then the trace file can be quite large. On the other hand, the trace file only specifies the propagation between node pairs when the propagation changes; hence, if nodes move slowly, then the propagation trace file is quite 

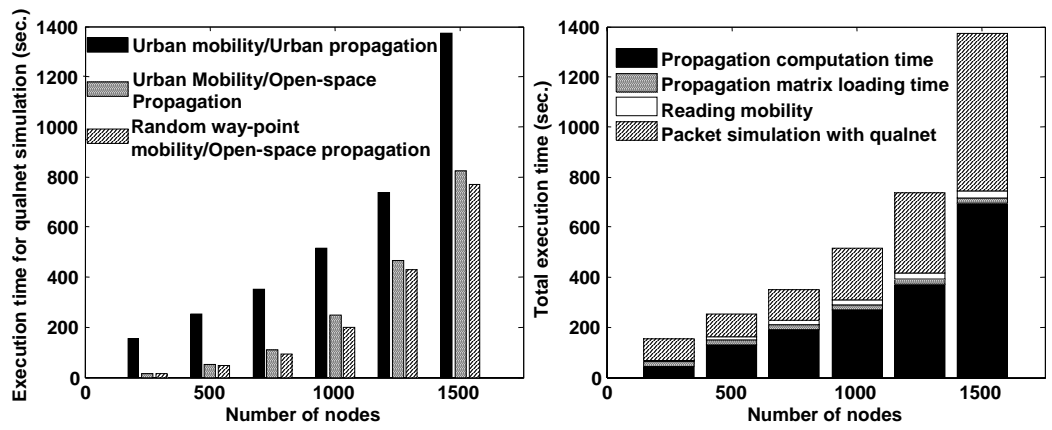

Fig. 16. Simulation Execution Time. The left-hand frame shows the simulation execution time for three simulation techniques. The right-hand plot shows a break-down of the execution time when realistic propagation is included. The propagation computation time includes the time to identify which floor tile a node is nearest and the time to look-up the propagation between nodes from the propagation matrix.

small. Moreover, the trace file only provides the propagation once every second ${ }^{5}$. Therefore, if high data rates are achieved, then the computational complexity of processing packet transmission/reception events is higher than the computational complexity of loading the propagation matrix. Thus, when the propagation trace file is precomputed, the impact of including realistic propagation might be relatively small. In the right-hand frame of Figure 16, it can be seen that the simulation time grows slowly with the number of nodes. More specifically, in the worst-case, the propagation trace file grows quadratically with the number of nodes. However, Figure 16 shows that the simulation time does not grow quadratically except for 1500 nodes, which shows a large jump in simulation time. Interestingly, at this node density, the simulation with realistic propagation takes approximately as long as the simulations with open-space propagation.

\section{FUTURE WORK}

While the propagation simulation discussed above is significantly more realistic than the 2-ray model commonly used in mobile wireless network simulation, there are several areas that require further effort. For example, as discussed in Section III-G, propagation is time-varying, and is linked to the mobility of objects, specifically people and vehicles. Since the integrated mobility and propagation simulator include the locations of people and vehicles, these locations can be included into the propagation calculations. However, more effort is required to model the impact of people and other mobile objects. One approach is to incorporate stochastic models. In this case, the simulation strategy would be site-specific and mixed deterministic and stochastic. See [32] for mixed deterministic and stochastic propagation modeling.

\footnotetext{
${ }^{5}$ Currently, iinear interpolate is used to determine the propagation between updates.
} 
Modeling propagation from indoors to outdoors and visa versa is an area of ongoing research [33]. One difficulty is that accurate propagation through non homogeneous walls is difficult to compute. As shown in Sections III-C and III-D, propagation is greatly influenced by the material. However, these sections only considered propagation through walls made from a single material, whereas most walls are made of several types of material. While [34] develops a technique to model propagation through complicated walls, it is computationally complex. Furthermore, even if more computational power is available, there remains the difficulty of determining the materials and wall structure of realistic urban buildings.

Throughout the discussion and in the computations above, it was assumed that transmitter and receiver antennas are vertically polarized. Furthermore, we also assumed that they are ideal. While it is straightforward to include the model of an ideal dipole antenna, in many cases the antenna is not an ideal dipole and is not vertically positioned. For example, see [35] for an example where the angle of inclination the antenna played a critical role in connectivity.

\section{RELATED WORK}

Currently, free-space and two-ray propagation are the most popular propagation models for MANETs research. For example, ns-2 [36], [37] only supports free-space and 2-ray propagation models. On the other hand, QualNet [38] supports open-space propagation as well as stochastic propagation models such as Rayleigh, Rician and Lognormal fading. Qualnet also supports channel gain trace files. The UDelModels simulation package generates trace files that are compatible with Qualnet. Furthermore, OPNET [39] supports open-space propagation models as well as an enhanced open-space model that accounts for hills, foliage and atmospheric affects.

There has been little effort on integrating realistic propagation into mobile wireless network simulation. Some examples where propagation modeling is considered include [40], [41], [42], and [43] where obstacles were included in the simulated environment and propagation was limited to line-of-sight. In [41], the obstacles were randomly placed buildings. But these have limited applicability because, as is shown in the Table I, most of the communication in an urban area is non line-of-sight. Other examples of propagation simulation in MANETs are [44], where raytracing is used to enhance ns-2's propagation model, and [45], where indoor MANET simulations make use of the attenuation factor model similar to what is discussed in Section IV-C.

Within the communications area, propagation prediction continues to be an active area of research. Ray-tracing (e.g., [46], [47]) and the vertical plane method [25] discussed in Section IV-B are often used. See [33] for a detailed 
review of work in propagation prediction.

There are several commercial packages that can be used to predict coverage of a single or a small number of mobile phone base stations or wireless access points (examples include [48], [49] and [50]). These tools have limited applicability to LUMNet simulation. Specifically, due to different goals (realistic vs. prediction), propagation predictions are typically more computationally intensive than is required for realistic network simulation. Furthermore, many tools focus on outdoor coverage for mobile phones, or indoor coverage for wireless base stations; they neglect mixed indoor/outdoor simulation scenarios.

\section{CONCLUSION}

Issues related to propagation simulation for urban wireless networking have been explored. Also, the design of a propagation simulator has been discussed. This propagation simulator is integrated with a mobility simulator discussed in [30]. This simulation package, which is available for download [5], demonstrates that it is possible to include realistic propagation into simulation of urban wireless networks such as the ones being deployed in several cities. As has been shown, traditional simulators that use random way-point mobility and the 2-ray propagation model are not suitable for urban environments. It is important to note that the simulation strategies discussed here and implemented in the simulation tools focus on realistic simulation, not accurate prediction. Thus, they are suitable for understanding the performance of urban wireless networking protocols, but not for planning specific networks. However, as computational resources increase, we expect that more complicated and accurate techniques will be integrated into the simulation packages, and will, perhaps, allow for accurate prediction as well.

This paper only provide details on realistic propagation of LUMNets. It does not describe realistic mobility and nor how to simulate the relevant application traffic.

\section{REFERENCES}

[1] Philadelphia Mayor’s Office, “Government leaders teleconference - wireless: The 21stCentury techhology,” 2004. [Online]. Available: http://www.phila.gov/mois/press/ multimedia/pdfs/Wireless Philadelphia092004.pdf

[2] City and C. of San Francisco, "Request for proposals 2005-19 - TechConnect: Community wireless broadband network,” Available at http://www.sfgov.org/ site/ tech connect index.asp, 2005.

[3] T. Times, "Ma says taipei to be wireless in two days,” December 19, 2005.

[4] T. C. of Minneapolis, "Request for proposal to provide broadband IP data access services," 2005.

[5] S. Bohacek, V. Sridhara, and J. Kim, "UDel models," http://udelmodels.eecis.udel.edu. 
[6] M. Hata, "Empirical formula for propagation loss in land mobile radio services," IEEE transactions on vehicle technology, vol. 29, no. 3, pp. 317-325, 1980.

[7] European Cooperation in the Field of Scientific and Technical Research, "Urban transmission loss models for mobile radio in 900 and 1800 MHz bands,” The Hague, Tech. Rep. Revision 2, 1991.

[8] T. Rappaport, Wireless Communications - Principles and Practice. Prentice Hall, 2002.

[9] F. Graziosi and F. Santucci, “A general correlation model for shadow fading in mobile radio systems,” IEEE Communication Letters, vol. 3, pp. 102-104, 2002.

[10] M. Gudmundson, “Correlation model for shadow fading in mobile radio systems,” Electron. Lett., vol. 27, pp. 2145-2146, 1991.

[11] H. L. Bertoni, Radio Propagation for Modern Wireless Systems. New Jersey: Prentice Hall, 1999.

[12] C. A. Balanis, Advanced Engineering Electromagnetics. New York: Wiley, 1989.

[13] D. Aguayo, J. Bicket, S. Biswas, G. Judd, and R. Morris, "Link-level measurements from an 802.11b mesh network," in Proceedings of the 2004 Conference on Applications, Technologies, Architectures, and Protocols for Computer Communications (SIGCOMM). New York: ACM Press, 2004, pp. 121-132.

[14] M. Yarvis, K. Papagiannaki, and W. S. Conner, "Characterization of 802.11 wireless networks in the home," in 1st Workshop on Wireless Network Measurements (WiNMee), 2005.

[15] S. V. Savov and M. H. A. J. Herben, "Modal transmission-line modeling of propagation of plane radiowaves through multilayer periodic building structures,” IEEE Transactions on antennas and propagation, vol. 51, no. 9, pp. 2244-2251, 2003.

[16] R. G. Kouyoumjian and P. H. Pathak, "A uniform geometrical theory of diffraction for an edge in a perfectly conducting surface," Proceedings of the IEEE, vol. 62, no. 11, pp. 1448-1461, 1974.

[17] J. B. Andersen, “UTD multiple-edge transition zone diffraction,” IEEE Transactions on Antennas and Propagation, vol. 45, pp. 1093-1097, 1997.

[18] K. Rizk, J. Wagen, J. Li, and F. Gardiol, "Lamppost and panel scattering compared to building reflection and diffraction,” in COST 259 TD, Turin, Italy, 1996, pp. 158-167.

[19] D. M. L. Vinc and M. A. Karam, "Dependence of attenuation in a vegetation canopy on freqency and plant water content," IEEE Transactions on Geoscience and Remote Sensing, vol. 34, no. 5, pp. 1090-1096, 1996.

[20] T. Kurner and A. Meier, "Prediction of outdoor and outdoor-to-indoor coverage in urban areas at 1.8 GHz." IEEE Jourbal on Selected Areas in communication, vol. 20, no. 3, pp. 496-506, 2002.

[21] V. Sridhara, H.-C. Shin, and S. Bohacek, “Observations and models of time-varying channel gain in crowded areas,” in 2nd International Workshop on Wireless Network Measurement, WinMee '06, Boston, Massachusetts, USA, 2006.

[22] C. Steger, P. Radosavljevic, and J. P. Frantz, “Performance of IEEE 802.11b wireless LAN in an emulated mobile channel,” in The 57th IEEE Semiannual Vehicular Technology Conference (VTC 2003-Spring), 2003, pp. 22-25.

[23] L. J. Greenstein, V. Erceg, and Y. S. Y. M. V. Clark, “A new path-gain/delay-spread propagation model for digital cellular channels,” IEEE Transactions on Vehicular Technology, vol. 46, no. 2, pp. 477-485, 1997.

[24] M. V. Clark, K. K. Leung, B. McNair, and Z. Kostic, “Outdoor IEEE 802.11 cellular networks: Radio link performance,” in IEEE International Conference on Communications (ICC). New York, NY: IEEE, 2002, pp. 595-599. 
[25] G. Liang and H. L. Bertoni, “A new approach to 3-d ray tracing for propagation prediction in citiee,” IEEE transaction on Antennas and propagation, vol. 46, pp. 853-863, 1998.

[26] K. Rizk, R. Valenzuela, S. Fortune, D. Chizhik, and F. Gardiol, "Lateral, full-3d and vertical plane propagation in microcells and small cells," in 48th IEEE Vehicle Technology Conference, 1998, pp. 998-1002.

[27] Berkeley Varitronics, “http://www.bvsystems.com/.”

[28] K. Rizk, J. F. Wagen, and F. Gardiol, "Influence of database accuracy on two-dimensional ray-tracing-based predictions in urban microcells,” IEEE Transactions on Vehicle Technology, vol. 49, pp. 631-642, 2000.

[29] K. Rizk, J. Wagen, and F. Gardiol, “Two-dimensional ray-tracing modeling for propagation in microcellular environments,” IEEE Transaction of Vehicle Technology, vol. 46, pp. 508-517, 1997.

[30] J. Kim and S. Bohacek, “A survey-based mobility model of people for simulation of urban mesh networks," in MeshNets, 2005 (available at http://www.eecis.udel.edu/ bohacek).

[31] C. E. Perkins and E. M. Royer, “Ad hoc on-demand distance vector routing,” Proceedings of the 2ns IEEE Workshop on Mobile Computing Systems and Applications, pp. 90-100, 1999.

[32] A. Domazetovic, L. J. Greenstein, N. B. Mandayam, and I. Seskar, "Propagation models for short range wireless channels with predictable path geometries,” IEEE Transactions on Communications, vol. 53, no. 7, pp. 1123-1126, 2005.

[33] M. F. Iskander and Y. Zhengqing, “Propagation prediction models for wireless communication systems,” IEEE Transacations on Microwave Theory and Techniques, vol. 50, no. 3, pp. 662-673, 2002.

[34] C. L. Holloway, P. L. Perini, R. R. DeLyser, and K. C. Allen, "Analysis of composite walls and their effects on short-path propagation modeling,” IEEE transactions on Vehicle Technology, vol. 46, pp. 730-738, 1997.

[35] S. Jadhav, T. X. Brown, S. Doshi, D. Henkel, and R. G.Thekkekunnel, “Lessons learned constructing a wireless ad hoc network test bed,” 1st International Workshop on Wireless Network Measurements, 2005.

[36] “Network Simulator - ns-2, http://www.isi.edu/nsnam/ns/.”

[37] “Wireless and mobility extensions to ns-2 http://www.monarch.cs.cmu.edu/cmu-ns.html.”

[38] Scalable Network Technologies, “The QualNet simulator http://www.qualnet.com/.”

[39] OPNET, "http://www.opnet.com."

[40] P. Johansson, T. Larsson, N. Hedman, B. Mielczarek, and M. Degermark, "Scenario-based performance analysis of routing protocols for mobile ad-hoc networks,” in MobiCom, 1999.

[41] A. Jardosh, E. M. Belding-Royer, K. C. Almeroth, and S. Suri, “Towards realistic mobility models for mobile ad hoc networks,” in MobiCom, 2003.

[42] A. P. Jardosh, E. M. Belding-Royer, K. C. Almeroth, and S. Suri, "Real-world environment models for mobile network evaluation,” IEEE Journal on Selected Areas in Communications, vol. 23, no. 3, pp. 622-632, 2005.

[43] S. Marinoni and H. H. Kari, "Ad hoc routing protocol performance in a realistic environment," in Proceedings of the Fifth IEEE International Conference on Networking (ICN 2006). Conference Publishing Services, to appear.

[44] J.-M. Dricot and P. D. Doncker, "High-accuracy physical layer model for wireless network simulations in NS-2,” in IWWAN, 2004.

[45] A. L. Cavilla, G. Baron, T. E. H. A. L. Litty, and E. de Lara, "Simplified simulation models for indoor MANET evaluation are not robust," in Proc. SECON, 2004. 
[46] R. A. Valenzuela and L. J. Greenstein, "Performance evaluations for urban line-of-sight microcells at $900 \mathrm{MHz}$ using a multi-ray propagation model,” in Global Telecommunications Conference GLOBECOM, 1991, pp. 1947-1952.

[47] S. Y. Seidel and T. S. Rappaport, "A ray tracing technique to predict path loss and delay spread inside buildings,” in Global Telecommunications Conference, 1992, pp. 649-653.

[48] WirelessValley, "LANPlanner http://www.wirelessvalley.com.”

[49] S. J. Fortune, D. M. Gay, B. W. Kernighan, O. Landron, R. A. Valenzuela, and M. H.Wright, "WISE design of indoor wireless systems: Practical computation and optimization,” Computational Science and Engineering, vol. 2, no. 1, pp. 58-68, 1995.

[50] WaveCall, "WaveSight http://www.wavecall.com/.” 